\title{
CONSTRAINING THE ORIGIN OF TeV PHOTONS FROM GAMMA-RAY BURSTS WITH DELAYED MeV-GeV EMISSION FORMED BY INTERACTION WITH COSMIC INFRARED/MICROWAVE BACKGROUND PHOTONS
}

\author{
X. Y. WANG, ${ }^{1,2}$ K. S. CHENG, ${ }^{2}$ Z. G. DAI, ${ }^{1}$ AND T. $\mathrm{Lu}^{3}$ \\ Received 2003 September 13; accepted 2003 November 17
}

\begin{abstract}
It has been suggested that electromagnetic cascade of very high-energy gamma-rays from gamma-ray bursts (GRBs) in the infrared/microwave background can produce delayed $\mathrm{MeV}-\mathrm{GeV}$ photons. This delay could be caused by the angular spreading effect of the scattered microwave photons or deflection of the secondary pairs due to the intergalactic magnetic field. Very high-energy $\mathrm{TeV}$ photons of GRBs could be produced by a few mechanisms including the proton-synchrotron radiation and electron inverse Compton emission from GRB internal shocks as well as external shocks. We suggest that the information provided by the delayed emission could give constraints on models for $\mathrm{TeV}$ gamma-rays. A more accurate calculation of the delayed time caused by the angular spreading effect is presented by considering recent observations of the extragalactic infrared background and the theoretical high-redshift infrared background. We also suggest that the dependence of the maximum time delay of scattered photons on their energies, if determined by future GLAST detector, could differentiate the two mechanisms causing the time delay.
\end{abstract}

Subject headings: diffuse radiation — gamma rays: bursts — magnetic fields

\section{INTRODUCTION}

Giga-electron volt emission from gamma-ray burst (GRB) sources is now considered a well-established fact (e.g., Sommer et al. 1994; Hurley et al. 1994) and there is also tentative evidence for $\mathrm{TeV}$ emission (e.g., Amenomori et al. 1996; Padilla et al. 1998). Recently, the Milagro group reported the detection of an excess of TeV gamma-rays above the background from one of the 54 BATSE GRBs (GRB 970417a) in the field of view of their detector, with a chance probability $\sim 1.5 \times 10^{-3}$ (Atkins et al. 2000). Poirier et al. (2003) reported suggestive evidence for sub-TeV gamma rays arriving in coincidence with GRBs. Although these observations were not claimed as firm detection, the production of $\mathrm{TeV}$ photons are also predicted by GRB theories. The emission mechanism for $\mathrm{TeV}$ photons includes electron inverse Compton (IC) emission and synchrotron emission from the protons accelerated by GRB shocks. ${ }^{4}$ The shocks could be internal shocks, external forward shocks, or external reverse shocks of GRBs. Such very highenergy photons at cosmological distance, however, may largely be absorbed by interacting with the cosmic infrared background radiation (CIB; e.g., Stecker, De Jager, \& Salamon 1992; Madau \& Phinney 1996). The IC scattering of the created $e^{+} e^{-}$ pairs off the cosmic microwave background (CMB) photons will produce delayed $\mathrm{MeV}-\mathrm{GeV}$ emission (Cheng \& Cheng 1996; Dai \& Lu 2002). There are two likely mechanisms causing the time delay. One is the angular spreading effect of the secondary pairs, i.e., the scattered microwave photons

\footnotetext{
${ }^{1}$ Department of Astronomy, Nanjing University, Nanjing 210093, China. 2 Department of Physics, University of Hong Kong, Hong Kong, China.

3 Purple Mountain Observatory, Chinese Academy of Sciences, Nanjing 210008, China.

${ }^{4}$ The decay of $\pi^{0}$ produced in photo-meson interactions in internal (Waxman \& Bahcall 1997) or external shocks (Waxman \& Bahcall 2000; Dai $\& \mathrm{Lu} 2001$ ) of GRBs could also lead to production of very high-energy photons, but with characteristic energies higher than $10^{14} \mathrm{eV}$. So a low radiation efficiency of $\mathrm{TeV}$ photons is expected for this process.
}

deviate from the direction of the original $\mathrm{TeV}$ photons by an angle $\sim 1 / \gamma$, where $\gamma$ is the Lorentz factor of the $e^{+} e^{-}$pairs (Cheng \& Cheng 1996; Dai \& Lu 2002). Another mechanism is related to the deflection of the propagating direction of the pairs in the intergalactic magnetic field (IGMF), if this field is sufficiently strong (Plaga 1995).

Dai \& $\mathrm{Lu}(2002)$ have discussed the spectrum and duration of the delayed emission, assuming that the high-energy primary photons $\left(\mathcal{E}_{\gamma}>300 \mathrm{GeV}\right)^{5}$ are produced by the electron IC emission in internal shocks. Recently, Guetta \& Granot (2003) argued that the intrinsic cutoff energy of photons from internal shocks can hardly extend to $\gtrsim 100 \mathrm{GeV}$ for typical GRBs with peak energy in the BATSE energy range. Here we extend the work of Dai \& Lu (2002) by considering that TeV photons could also come from external shocks and could be produced by proton-synchrotron radiation. We further suggest that the information provided by the delayed emission could constrain the emission mechanism of the $\mathrm{TeV}$ photons from GRBs and distinguish between the two mechanisms causing the time delay. We will also study the detectablity of the delayed emission by the future GLAST detector.

In $\S 2$, we present three emission processes for $\mathrm{TeV}$ photons and the corresponding spectrum and calculate the cutoff energy for high-energy photons from external shocks due to $\gamma-\gamma$ pair attenuation with softer photons. We find that the cutoff energy exceeds $10 \mathrm{TeV}$ for typical parameters. In $\S 3$, we compare the spectra of the delayed emission for these three emission models of $\mathrm{TeV}$ photons and give a more accurate calculation of the delayed time caused by the angular spreading by considering recent observations of the extragalactic infrared background and the theoretical high-redshift infrared background. Then, in $\S 4$, we study the particular case of GRB 940217, from which delayed emission had already

\footnotetext{
${ }^{5}$ Please note that here $\mathcal{E}_{\gamma}$ is the gamma-ray energy at the rest frame of GRBs.
} 
been detected by EGRET. Finally, we give the conclusions and discussions.

\section{TeV EMISSION MODELS OF GAMMA-RAY BURSTS}

\subsection{Cutoff Energy of High-Energy Photons from External Shocks}

GRBs are thought to be caused by the dissipation, through shocks, of the kinetic energy of a relativistically expanding fireball with a Lorentz factor $\Gamma_{0} \sim 10^{2}-10^{3}$. The shocks could be either internal (Paczyński \& Xu 1994; Rees \& Mészáros 1994) due to collisions between fireball shells or external (Rees \& Mészáros 1992; Dermer, Böttcher, \& Chiang 1999) due to the interaction of the fireball with the external medium. When the relativistic ejecta encounters the external medium, a relativistic forward shock expands into the external medium, and a reverse shock moves into and heats the fireball ejecta (Sari \& Piran 1999). Very high-energy photons can be produced by electron IC and proton-synchrotron emission in both internal shocks and external shocks. ${ }^{6}$ There should be a cutoff in the high-energy gamma-ray spectrum due to the internal absorption of high-energy gamma rays by pair-production in GRBs. (e.g., Baring \& Harding 1997; Totani 1999; Lithwick \& Sari 2001) Recently, Guetta \& Granot (2003) argue that a high cutoff energy for emission from internal shocks needs large value of the initial Lorentz factor $\Gamma_{0}$ or variability time $t_{v}$, but at the same time, they imply lower values of the peak energy $E_{p}$ of the synchrotron emission. So they conclude that $\mathrm{TeV}$ photons from internal shocks can hardly reconcile with typical GRBs but may be related with X-ray flashes. However, this conclusion is dependent on the assumption that the observed GRB spectral peak is due to characteristic synchrotron photon energy, but this is not completely confirmed. For example, Totani (1999) pointed out that efficient pair-production in GRBs may affect the peak of GRB spectrum around MeV. We note that $\mathrm{TeV}$ photons could also come from external shocks and the cutoff energy may be significantly increased because external shocks have much larger sizes than those of internal shocks. Below we will first estimate the cutoff energy for external shocks.

We adopt an analytical approach similar to the one developed for internal shocks by Lithwick \& Sari (2001) and applied to afterglows by Zhang \& Mészáros (2001), but we here apply to the initial phase of external shocks. The attenuation optical depth of high-energy photons with softer photons is (Lithwick \& Sari 2001)

$$
\tau_{\gamma \gamma}=(11 / 180) \sigma_{\mathrm{T}} N\left(>E_{\mathrm{an}}\right) / 4 \pi r_{\mathrm{dec}}^{2},
$$

where $N\left(>E_{\text {an }}\right)$ is the total photon number with energy above the attenuation threshold energy $E_{\mathrm{an}}, r_{\mathrm{dec}}$ is the deceleration radius at which external shooks take place, and $\sigma_{\mathrm{T}}$ is the Thomson cross section. Like Zhang \& Mészáros (2001), we assume that the emission spectrum around $E_{\text {an }}=h \nu_{\text {an }}$ is $L(\nu)=F_{\nu} 4 \pi D^{2} /(1+z) \propto \nu^{-\beta}$, then the optical depth is

$$
\tau_{\gamma \gamma}=\frac{(11 / 180) \sigma_{\mathrm{T}} F_{\nu}\left(\nu_{\mathrm{an}}\right) d_{L}^{2}}{4 \Gamma_{0}^{4} c^{2} h \beta t_{\mathrm{dec}}}
$$

where $d_{L}$ is the source distance, $z$ is the redshift of the source, $h$ is Planck's constant, and $t_{\mathrm{dec}}=10 E_{53}^{1 / 3} n_{0}^{-1 / 3}\left(\Gamma_{0} / 300\right)^{-8 / 3} \mathrm{~s}$

\footnotetext{
${ }^{6}$ Here the "external shock" used in this paper means the shock at the initial phase of the deceleration of the fireball, not the later afterglow shock.
}

is the deceleration timescale of the GRB ejecta. For primary photons in the $\mathrm{TeV}$ band, the attenuation threshold energy is $E_{\text {an }}=h \nu_{\text {an }}=20\left(\Gamma_{0} / 300\right)^{2}\left(\mathcal{E}_{\gamma} / 1 \mathrm{TeV}\right)^{-1} \mathrm{keV}$. At $h \nu \sim$ $10 \mathrm{keV}$, the emission is dominated by the electron synchrotron radiation from the external forward shocks (see Fig. 2 in Wang, Dai, \& Lu 2001). The two characteristic frequencies and the peak flux of the synchrotron spectrum of the external forward shocks are given by

$$
\begin{gathered}
\nu_{m}^{\mathrm{fs}}=4 \times 10^{20}\left(\frac{p-2}{p-1}\right)^{2}\left(\frac{\epsilon_{e}}{0.5}\right)^{2} \epsilon_{B,-2}^{1 / 2}\left(\frac{\Gamma_{0}}{300}\right)^{4} n_{0}^{1 / 2} \mathrm{~Hz}, \\
\nu_{c}^{\mathrm{fs}}=\frac{10^{17}}{(Y+1)^{2}} E_{53}^{-1 / 2} \epsilon_{B,-2}^{-3 / 2} n_{0}^{-1}\left(\frac{t_{\mathrm{dec}}}{10 \mathrm{~s}}\right)^{-1 / 2} \mathrm{~Hz},
\end{gathered}
$$

and

$$
F_{\nu_{m}}^{\mathrm{fs}}=26 D_{L, 28}^{-2} \epsilon_{B,-2}^{1 / 2} E_{53} n_{0}^{1 / 2} \mathrm{mJy},
$$

respectively, where $\epsilon_{e}$ and $\epsilon_{B}$ are the fractions of the shock energy carried by electrons and magnetic field, respectively, $Y \simeq\left(\epsilon_{e} / \epsilon_{B}\right)^{1 / 2}$ is the Compton factor, and $n$ is the number density of the external medium. We use the usual notation $a=10^{n} a_{n}$ throughout the paper. Generally, we have $\nu_{c}^{\mathrm{fs}}<\nu_{\mathrm{an}}<\nu_{m}^{\mathrm{fs}}$, so

$$
\begin{aligned}
F_{\nu}\left(\nu_{\mathrm{an}}\right)= & \left(\frac{\nu_{\mathrm{an}}}{\nu_{c}^{\mathrm{fs}}}\right)^{-1 / 2} F_{\nu_{m}}^{\mathrm{fs}}=3.8(Y+1)^{-1}\left(\frac{\Gamma_{0}}{300}\right)^{-1} \\
& \times E_{53}^{3 / 4} \epsilon_{B,-2}^{-1 / 4} n_{0}^{1 / 2} t_{\mathrm{dec}, 1}^{-1 / 4} D_{L, 28}^{-2}\left(\frac{\mathcal{E}_{\gamma}}{1 \mathrm{TeV}}\right)^{1 / 2} \mathrm{mJy} .
\end{aligned}
$$

From equation (1), finally we get the cutoff energy where $\tau_{\gamma \gamma}=1$,

$$
E_{\text {cut }} \sim 40\left(\frac{\Gamma_{0}}{300}\right)^{10 / 3} E_{53}^{-2 / 3} \epsilon_{B,-2}^{1 / 2} n_{0}^{-5 / 6} \mathrm{TeV} .
$$

\subsection{Spectrum of the Primary $\mathrm{TeV}$ Photons for Different Emission Models}

One mechanism for the $\mathrm{TeV}$ photon production from GRBs is the electron IC emission in GRB shocks. As the electrons in internal shocks and external forward shocks are in the fastcooling regime and $h \nu_{\mathrm{KN}}^{\mathrm{IC}}<\mathrm{TeV}<h \nu_{M}^{\mathrm{IC}}$, the energy spectrum of the electron IC emission at $\mathrm{TeV}$ band can be commonly described as $\nu F_{\nu} \propto \nu^{-p+1 / 2}$ (Guetta \& Granot 2003), where $h \nu_{\mathrm{KN}}^{\mathrm{IC}}=\Gamma_{0}^{2} m_{e}^{2} c^{4} / h \nu_{m}=22 \mathrm{GeV}\left(\Gamma_{0} / 300\right)\left(h \nu_{m} / 1 \mathrm{MeV}\right)^{-1}\left(\nu_{m}\right.$ represents the peak frequency of the synchrotron spectrum for internal or forward shocks) and $h \nu_{M}^{\mathrm{IC}}=\Gamma_{0} \gamma_{M} m_{e} c^{2}\left(\gamma_{M}\right.$ is the maximum Lorentz factor of the electrons accelerated by shocks). On the other hand, for reverse shocks, $h \nu_{c}^{\text {IC }}<$ $\mathrm{TeV}<h \nu_{\mathrm{KN}}^{\mathrm{IC}}$ (where $\nu_{c}^{\mathrm{IC}} \simeq 2 \gamma_{c}^{2} \nu_{c}, \nu_{c}$ is the cooling break frequency of the reverse shocks and $\gamma_{c}$ is the Lorentz factor of the corresponding electrons), so the $\mathrm{TeV}$ spectrum is given by $\nu F_{\nu} \propto \nu^{-p / 2+1}$ (Sari \& Esin 2001).

In the region where the electrons are accelerated, protons may be also accelerated up to ultrahigh energies of more than $10^{20} \mathrm{eV}$ (Waxman 1995; Vietri 1995), producing a spectrum characteristic of Fermi mechanism $d N_{p} / d E_{p} \propto E_{p}^{-p}$. The possibility of energetic protons, accelerated in both internal shocks and external shocks, producing $\sim \mathrm{TeV}$ gamma rays by 
synchrotron emission has been discussed by a number of authors (Vietri 1997; Böttcher \& Dermer 1998; Totani 1998a, 1998b). For external shocks, the postshock magnetic field is $B=\left(32 \pi \epsilon_{B} \Gamma_{0}^{2} n m_{p} c^{2}\right)^{1 / 2}$ and the energy of the synchrotron photons is given by

$$
E_{p \text {-syn }}=\frac{\Gamma_{0} \Gamma_{p}^{2} e h B}{2 \pi m_{p} c}=3 \mathrm{TeV} E_{p, 21}^{2} \epsilon_{B}^{1 / 2} n_{0}^{1 / 2}
$$

where $\Gamma_{p}$ and $E_{p}$ are, respectively, the Lorentz factor and energy of the protons. Totani (1998a, 1998b) argues that the protons can be accelerated up to $10^{20}-10^{21} \mathrm{eV}$ for $\Gamma_{0}=100-1000$, so we expect $E_{p \text {-syn }}$ can extend to TeV band for $\epsilon_{B} n \sim 1$. The energy spectrum from proton-synchrotron radiation is $\nu F_{\nu} \propto \nu^{(3-p) / 2}$, which is distinct from the electron IC emission spectrum we discussed above.

\section{SPECTRUM, DURATION, AND INTENSITY OF THE DELAYED EMISSION}

\subsection{Spectrum of the Delayed Emission}

Tera-electron volt gamma-rays emitted from extragalactic sources may collide with diffuse cosmic infrared background (CIB) photons, leading to secondary $e^{+} e^{-}$pairs. The pair production optical depth $\tau_{\gamma \gamma}$ depends on the spectral energy distribution and the intensity of the CIB, which is currently not well known. Because of the high redshift of cosmological GRB sources, $\tau_{\gamma \gamma}$ also depends on the evolution of the CIB with the redshift, which also remains uncertain. Despite these uncertainties, calculations based on the theoretical models (e.g., De Jager \& Stecker 2002; Aharonian et al. 2002) of CIB and modeling of observations of the TeV blazar H1426+428 (Aharonian et al. 2002; Costamante et al. 2003) show that $\tau_{\gamma \gamma}$ is significantly larger than unity for photons with $\mathcal{E}_{\gamma} \gtrsim$ $500 \mathrm{GeV}$ from extragalactic GRBs with redshift $z \gtrsim 0.3$ (also see Totani \& Takeuchi 2002, in which a somewhat lower optical depth is obtained). On the other hand, $\mathcal{E}_{\gamma}$ from the synchrotron radiation of protons or electron IC emission may extend to a few $\mathrm{TeV}$. So we here choose to study the primary very high-energy photons with energy in the range of $0.5-$ $5 \mathrm{TeV}$ that are almost totally absorbed by the CIB photons. We assume a general form for the energy spectrum of the primary high-energy emission at $\mathrm{TeV}$ band: $\nu L_{\nu} \propto \nu^{-\alpha}$. The photon spectrum is accordingly $N_{\nu} \propto \nu^{-(\alpha+2)}$, and the spectrum of the secondary pairs is then $d N_{e} / d \gamma_{e} \propto \gamma_{e}^{-(\alpha+2)}$. The secondary pairs would boost the CMB photons to higher energy by IC scattering. The scattered photons (or delayed photons) will have a characteristic energy

$$
\varepsilon=\frac{4}{3} \gamma_{e}^{2}\langle\epsilon\rangle=0.8\left(\frac{\mathcal{E}_{\gamma}}{1 \mathrm{TeV}}\right)^{2} \mathrm{GeV},
$$

where $\langle\epsilon\rangle=2.7 k T_{\mathrm{CMB}}$ is the mean energy of the CMB photons and $\gamma_{e}$ is the Lorentz factor of the secondary pairs resulted from a primary photon with energy $\mathcal{E}_{\gamma}$. So for $\mathcal{E}_{\gamma}$ in the range of $0.5-5 \mathrm{TeV}$, the energies of the scattered photons are in the range $200 \mathrm{MeV}-20 \mathrm{GeV}$ correspondingly.

The time integrated spectrum of the scattered CMB photons should be

$$
\frac{d N_{\varepsilon}}{d \varepsilon} \propto \varepsilon^{-(\alpha+4) / 2}
$$

(Blumenthal \& Gould 1970; Dai \& Lu 2002). Strictly speaking, this form holds only when all the $\mathrm{TeV}$ photons are absorbed locally, i.e., their production mean free path $R_{\text {pair }}$ should be much smaller than the luminosity distance of the sources; otherwise, $\mathrm{TeV}$ photons of different energy may be absorbed at different redshift $z_{\text {pair }}$, causing the observed delayed photons energy shifted from equation (9) by a factor $1-\left[\left(1+z_{\text {pair }}\right) /(1+z)\right]^{2}$. But, for the calculated values of $R_{\text {pair }}$ in the next subsection, we find that this factor is within $15 \%$ and so this form holds with a good approximation. It is important to note that if we choose the part of $\mathrm{TeV}$ photons that are totally absorbed locally, the spectrum of the delayed emission is independent of the poorly known CIB. For three different $\mathrm{TeV}$ models, the spectra of the scattered (delayed) emission are different, as presented in Table 1. From Table 1, we can see that the spectra of the delayed emission are significantly different from each other and we can therefore use this difference to constrain the emission mechanism of the primary $\mathrm{TeV}$ photons.

\subsection{The Duration of the Delayed Emission}

The duration of the scattered CMB photons should be the maximum of three timescales: $\tau_{1}$, the observed IC cooling life time of the secondary electrons; $\tau_{2}$, the timescale caused by the deflection of the electrons due to the IGMF; and $\tau_{3}$, the

TABLE 1

Comparison of the Spectra of the Primary TeV Photons and the Delayed Photons for Different Emission Models of TeV Photons

\begin{tabular}{ccc}
\hline \hline Model & $-(\alpha+2)$ (Values for $p=2.2)$ & $-\beta$ (Values for $p=2.2)$ \\
\hline Proton-synchrotron............................... & $-\frac{p+1}{2}(-1.6)$ & $-\frac{p+5}{4}(-1.8)$ \\
IC from reverse shocks......................... & $-\frac{p+2}{2}(-2.1)$ & $-\frac{p+6}{4}(-2.05)$ \\
IC from forward (or internal) shocks....... & $-\left(p+\frac{3}{2}\right)(-3.7)$ & $-\frac{2 p+7}{4}(-2.85)$ \\
\hline
\end{tabular}

Note.-The quantity $-(\alpha+2)\left[\left(d N_{\mathcal{E}_{\gamma}} / d \mathcal{E}_{\gamma}\right) \propto \mathcal{E}_{\gamma}^{-(\alpha+2)}\right]$ is the spectral index of the photon spectrum of the primary TeV photons, and $-\beta\left[\left(d N_{\varepsilon} / d \varepsilon\right) \propto \varepsilon^{-\beta}\right]$ is the spectral index of the photon spectrum of the delayed $\mathrm{MeV}-\mathrm{GeV}$ photons. 
angular spreading time (Dai \& Lu 2002; Dai et al. 2002). Dai $\& \mathrm{Lu}(2002)$ have derived

$\tau_{1}=\frac{3 m_{e} c}{8 \gamma_{e}^{3} \sigma_{\mathrm{T}} u_{\mathrm{CMB}}}=37\left(\frac{\mathcal{E}_{\gamma}}{1 \mathrm{TeV}}\right)^{-3} \mathrm{~s}=37\left(\frac{\varepsilon}{0.8 \mathrm{GeV}}\right)^{-3 / 2} \mathrm{~s}$,

where $u_{\mathrm{CMB}}$ is the energy density of the CMB photons and $\gamma_{e}$ is the Lorentz factor of the secondary electrons, and it relates to the energy of the primary $\mathrm{TeV}$ photons by $\gamma_{e}=$ $10^{6}\left(\mathcal{E}_{\gamma} / 1 \mathrm{TeV}\right)$. The timescale caused by the deflection of the electrons due to the IGMF is given by

$$
\begin{aligned}
\tau_{2} & =6.1 \times 10^{3}\left(\frac{\mathcal{E}_{\gamma}}{1 \mathrm{TeV}}\right)^{-5}\left(\frac{B_{\mathrm{IGMF}}}{10^{-20} \mathrm{G}}\right)^{2} \mathrm{~s} \\
& =6.1 \times 10^{3}\left(\frac{\varepsilon}{0.8 \mathrm{GeV}}\right)^{-5 / 2}\left(\frac{B_{\mathrm{IGMF}}}{10^{-20} \mathrm{G}}\right)^{2} \mathrm{~s} .
\end{aligned}
$$

To know $\tau_{3}$, we must know the mean free path $R_{\text {pair }}$ of the very high-energy photons in the extragalactic IR background, which depends on the intensity of the IR background. Electron-positron pair creation due to the interaction of a $\gamma$-ray photon of energy $\mathcal{E}_{\gamma}$ with a softer photon of energy $\epsilon$ can take place provided that $\mathcal{E}_{\gamma} \epsilon(1-\cos \theta) \geq 2\left(m_{e} c^{2}\right)^{2}$, where $\theta$ is the encounter angle of the two photons. For a fixed $\gamma$-ray energy $\mathcal{E}_{\gamma}$, the pair production cross section $\sigma$ rises steeply from the threshold $\epsilon_{\mathrm{th}}$, has a maximum value equal to $0.26 \sigma_{\mathrm{T}}$ at $\epsilon=$ $2 m_{e}^{2} c^{4} / \mathcal{E}_{\gamma}$ and then falls off as $\epsilon^{-1}$ for $\epsilon>\epsilon_{\mathrm{th}}$. Because of the peaked cross section, collisions will preferentially take place between $\gamma$-ray photons of energy $\mathcal{E}_{\gamma}$ and soft photons with energy $\sim 2 m_{e}^{2} c^{4} / \mathcal{E}_{\gamma}$. So for $\mathcal{E}_{\gamma}$ in the range of $0.5-5 \mathrm{TeV}$, the wavelengths of the softer photons with which pair production preferentially takes place are in the range $1.2-12 \mu \mathrm{m}$.

Since GRBs are at cosmological distances and CIB evolves with the redshift (Salamon \& Stecker 1998), $R_{\text {pair }}$ should depend on the redshift of the GRBs. For simplicity, we will discuss three representative cases with $(a) z=0.3,(b) z=1$, and $(c) z=3$.

Case $a(z=0.3)$. - It can be assumed that CIB is in place by a time corresponding to $z=0.3$, so the CIB photon number density at $z=0.3$ is $(1+z)^{3}$ higher than the value at $z=0$. Observations of CIB show that its spectral energy distribution has a peak around $1-2 \mu \mathrm{m}$ and a valley at mid-IR band. In the wavelength range of $\sim 1.2-12 \mu \mathrm{m}$ that we are interested in, the number densities of the CIB photons can be approximately shaped by $\bar{n}(\epsilon) \epsilon \propto \epsilon^{-k_{1}}$ with $-k_{1} \simeq 0$ (Coppi \& Aharonian 1999; Aharonian et al. 2002), which results in a nearly constant $R_{\text {pair }}$. The observed CIB flux $J_{\nu}$ at $2.2 \mu \mathrm{m}$ is of the order of $\sim 10 \mathrm{nW} \mathrm{m}{ }^{-2} \mathrm{sr}^{-1}$ (Wright \& Johnson 2001; Wright 2003), so the number density of the CIB photons at $z=0$ is

$$
\left.\bar{n}(\epsilon) \epsilon\right|_{\lambda=2.2 \mu \mathrm{m}}=\frac{4 \pi J_{\nu}}{c \epsilon}=0.45 \times 10^{-2} \mathrm{~cm}^{-3} .
$$

Then we obtain the mean free path for $\mathrm{TeV}$ photons at $z=0.3$ :

$R_{\text {pair }}=\frac{1}{0.26 \sigma_{\mathrm{T}} \bar{n}(\epsilon) \epsilon(1+z)^{3}}=0.55 \times 10^{27}\left(\frac{\mathcal{E}_{\gamma}}{1 \mathrm{TeV}}\right)^{-k_{1}} \mathrm{~cm}$, and

$$
\begin{aligned}
\tau_{3}(z=0.3) & =\frac{R_{\text {pair }}}{2 \gamma_{e}^{2} c}=1.0 \times 10^{4}\left(\frac{\mathcal{E}_{\gamma}}{1 \mathrm{TeV}}\right)^{-2-k_{1}} \mathrm{~s} \\
& =1.0 \times 10^{4}\left(\frac{\varepsilon}{0.8 \mathrm{GeV}}\right)^{-1-k_{1} / 2} \mathrm{s.}
\end{aligned}
$$

Case $b(z=1)$. - Salamon \& Stecker (1998) have derived the intergalactic comoving radiation energy density as a function of wavelength in the range $10^{-2}-2.5 \mu \mathrm{m}$ for several fixed redshifts by considering the stellar emissivity with and without metallicity. We shall assume that the power-law form $n(\epsilon) \epsilon \propto \epsilon^{-k_{2}}$ also holds in the range 1.2-12 $\mu \mathrm{m}$ for $z=1$, but the intensity of CIB is lower than that at $z \simeq 0$, according to the calculation result of Salamon \& Stecker (1998). At $\lambda=2.2 \mu \mathrm{m}$, the intergalactic comoving radiation energy density $U_{\nu}$ at $z=1$ is about $9 \times 10^{-30} \mathrm{ergs}^{-1} \mathrm{~cm}^{-3}$, so

$$
n(\epsilon, z=1) \epsilon=\frac{U_{\nu}}{h}=1.4 \times 10^{-3}\left(\frac{\lambda}{2.2 \mu \mathrm{m}}\right)^{k_{2}} .
$$

Finally, we obtain $R_{\text {pair }}$ and the delay time $\tau_{3}$ for bursts at $z=1$ :

$$
\begin{array}{r}
R_{\text {pair }}=\frac{1}{0.26 \sigma_{\mathrm{T}} n(\epsilon, z=1) \epsilon}=4 \times 10^{27}\left(\frac{\mathcal{E}_{\gamma}}{1 \mathrm{TeV}}\right)^{-k_{2}} \mathrm{~cm}, \\
\tau_{3}(z=1)=6.67 \times 10^{4}\left(\frac{\mathcal{E}_{\gamma}}{1 \mathrm{TeV}}\right)^{-2-k_{2}} \mathrm{~s} \\
=6.67 \times 10^{4}\left(\frac{\varepsilon}{0.8 \mathrm{GeV}}\right)^{-1-k_{2} / 2} \mathrm{~s}
\end{array}
$$

Case c $(z=3)$.- The CIB radiation energy density is even lower. At $\lambda=2.2 \mu \mathrm{m}, U_{\nu}$ is about $3 \times 10^{-30} \mathrm{ergs} \mathrm{Hz}^{-1} \mathrm{~cm}^{-3}$. So

$$
\begin{aligned}
\tau_{3}(z=3) & =2.2 \times 10^{5}\left(\frac{\mathcal{E}_{\gamma}}{1 \mathrm{TeV}}\right)^{-2-k_{3}} \mathrm{~s} \\
& =2.2 \times 10^{5}\left(\frac{\varepsilon}{0.8 \mathrm{GeV}}\right)^{-1-k_{3} / 2} \mathrm{~s}
\end{aligned}
$$

where $n(\epsilon) \epsilon \propto \epsilon^{-k_{3}}(\lambda=1.2-12 \mu \mathrm{m})$ for CIB at $z=3$. If we also consider the time dilation due to redshift, the delay time is even longer by a factor of $1+z$.

To know the values of $k_{2}$ and $k_{3}$ in the range $\lambda=1.2-12 \mu \mathrm{m}$, we need to know the intergalactic comoving radiation energy density beyond $\lambda=2.5 \mu \mathrm{m}$ (Salamon \& Stecker 1998). But we expect that the absolute value of $k_{2}$ or $k_{3}$ is small, since they reflect the characteristic shape of the starlight spectrum, which in the wavelength band between 1 and several microns behaves as $\nu I(\nu) \propto \lambda^{\beta}$ with $\beta \sim-1$.

Comparing these three timescales, we know that the observed IC life time $\tau_{1}$ is always much smaller than the other two timescales and therefore not related to the delay time. The time ${ }_{2}$ may be comparable to $\tau_{3}$ at $\varepsilon=0.8 \mathrm{GeV}$ if $B_{\mathrm{IGMF}} \sim 3 \times$ $10^{-20} \mathrm{G}$ for bursts at $z \simeq 1$. By now, very little is known about the IGMF. To interpret the observed $\sim \mu \mathrm{G}$ magnetic fields in galaxies and X-ray clusters, the seed fields required in dynamo theories could be as low as $10^{-20} \mathrm{G}$ (Kulsrud et al. 1997; 
Kulsrud 1999). Theoretical calculation of primordial magnetic fields show that these fields could be of order $10^{-20} \mathrm{G}$ or even as low as $10^{-29} \mathrm{G}$, generated during the cosmological QCD or electroweak phase transition, respectively (Sigl, Olinto, \& Jedamzik 1997). So, if we know which timescale is responsible for the delay time, we can constrain the strength of the IGMF.

A way to distinguish which mechanism causes the time delay $\Delta t$ is to examine the dependence of the maximum time delay $\Delta t(\varepsilon)$ of the scattered photons on their energy $\varepsilon$. From the expression of $\tau_{2}$ (eq. [12]) or $\tau_{3}$ (eqs. [15], [18], or [19]), we can obtain the dependence of the maximum time delay on photon energy. If the delay time is dominated by $\tau_{2}$, $\Delta t(\varepsilon) \propto \varepsilon^{-5 / 2}$, while $\Delta t(\varepsilon) \propto \varepsilon^{-(1+\kappa)}$ if it is dominated by $\tau_{3}$, where $\kappa=0, k_{1} / 2, k_{2} / 2$ for bursts at $z=0.3,1,3$, respectively.

Regardless which of the two timescales is responsible for the time delay, from the expressions of $\tau_{2}$ and $\tau_{3}$, we know that softer photons tend to have larger amounts of delay. Another feature characteristic of our model for the delayed emission is that the flux of the delayed emission is roughly a constant over the whole duration. These may be used to tell our model from other models for the delayed emission from GRBs. For example, Zhang \& Mészáros (2001) proposed that the electron IC emission from afterglow shocks could produce a delayed $\mathrm{GeV}$ component. But in their model, the GeV flux rises first to a peak and then declines in a power-law manner as $F_{\nu} \propto t^{(11-9 p) / 8}$ (Zhang \& Mészáros 2001).

\subsection{Intensity of the Delayed Emission}

We assume that the burst energy in the TeV energy range from $\mathcal{E}_{\gamma, 1}=h \nu_{1}=0.5 \mathrm{TeV}$ to $\mathcal{E}_{\gamma, 2}=h \nu_{2}=5 \mathrm{TeV}$ is a fraction $f$ of the burst energy in the BATSE energy range $E_{\mathrm{B}}$, i.e., $\int_{\nu_{1}}^{\nu_{2}} L_{\nu} d \nu \Delta t=f E_{\mathrm{B}}$, where $L_{\nu}$ is the luminosity per frequency and $\Delta t$ is the duration of the primary $\mathrm{TeV}$ emission, which is considered to be comparable to the prompt GRB duration in the BATSE band.

The energy of the primary photons from $h \nu_{1}$ to $h \nu_{2}$ is essentially redistributed to the scattered photons from $\varepsilon_{1}=$ $200 \mathrm{MeV}$ to $\varepsilon_{2}=20 \mathrm{GeV}$, i.e.,

$$
\int_{\varepsilon_{1}}^{\varepsilon_{2}} \varepsilon \frac{d N_{\varepsilon}}{d \varepsilon}=\int_{\nu_{1}}^{\nu_{2}} L_{\nu} d \nu \Delta t=f E_{\mathrm{B}} .
$$

So the observed, time integrated (over the total duration) differential energy spectrum is

$$
\begin{aligned}
\varepsilon^{2} \frac{d N_{\varepsilon}}{d \varepsilon} \Delta t= & \frac{\alpha}{2\left[1-\left(\nu_{2} / \nu_{1}\right)^{-\alpha}\right]} \frac{f E_{\mathrm{B}}}{4 \pi d_{L}^{2}}\left(\frac{\varepsilon}{\varepsilon_{1}}\right)^{-\alpha / 2} \\
= & f_{1} f \frac{f E_{\mathrm{B}}}{4 \pi d_{L}^{2}}\left(\frac{\varepsilon}{\varepsilon_{1}}\right)^{-\alpha / 2} \\
= & 6 \times 10^{-7} \cdot 11^{-\alpha / 2}\left(\frac{f_{1}}{0.25}\right) \\
& \times f_{-2} E_{\mathrm{B}, 53} d_{L, 28}^{-2}\left(\frac{\varepsilon}{0.8 \mathrm{GeV}}\right)^{-\alpha / 2} \mathrm{ergs} \mathrm{cm}^{-2},
\end{aligned}
$$

where $f_{1}=\alpha\left\{2\left[1-\left(\nu_{2} / \nu_{1}\right)^{-\alpha}\right]\right\}^{-1}$ is, respectively, 0.13, 0.25, and 0.85 for three kinds of $\mathrm{TeV}$ spectrum for $p=2.2$. The fraction $f$ of the burst energy in the TeV band is an unknown factor and may depend on the emission model and many unknown parameters like $\epsilon_{e}$ and $\epsilon_{B}$. For the model of the IC emission from the reverse shocks, we estimate $f \sim 0.01$ for typical shock parameters (see Fig. 2 in Wang et al. 2001), which is consistent with the observational result that the energy in the observed delayed emission from GRB 940217 is about a factor of 0.01 of the burst energy in the BATSE range.

The EGRET detector has fluence threshold of $\sim 2.1 \times$ $10^{-6}$ ergs $\mathrm{cm}^{-2}$ for a short integration time regime $(t<1.7 \times$ $\left.10^{3} \mathrm{~s}\right)$ and of $\sim 2.1 \times 10^{-6} \mathrm{ergs} \mathrm{cm}^{-2}\left(t / 1.7 \times 10^{3} \mathrm{~s}\right)^{1 / 2}$ for long-term observations. Therefore, EGRET could detect delayed $\mathrm{GeV}$ emission only from strong GRBs, such as GRB 940217. However, the future detector GLAST is much more sensitive. The fluence threshold for GLAST is roughly $\sim 4 \times 10^{-7}\left(t / 10^{5} \mathrm{~s}\right)^{1 / 2}$ ergs $\mathrm{cm}^{-2}$ for a long integration time regime (exposure time $t \gtrsim 10^{5} \mathrm{~s}$ ) and $\sim 4 \times 10^{-7} \mathrm{ergs} \mathrm{cm}^{-2}$ for a short integration time (Gehrels \& Michelson 1999; Zhang \& Mészáros 2001). So we expect that GLAST could detect delayed emission from typical GRBs with $E_{\mathrm{B}}=10^{53} \mathrm{ergs}$ at $d_{L}=10^{28} \mathrm{~cm}$.

\section{GRB 940217}

GRB 940217 is a very strong burst with a total fluence above $20 \mathrm{keV}$ of $(6.6 \pm 2.7) \times 10^{-4} \mathrm{ergs}^{-2}$ and a duration of $\sim 180 \mathrm{~s}$ in the BATSE range (Hurley et al. 1994). It has the third largest fluence of $\sim 800$ BATSE bursts up to the detection time of this burst. During the period of the low-energy emission, i.e., the first $\sim 180 \mathrm{~s}$, EGRET detected 10 photons with energies ranging from a few tens of $\mathrm{MeV}$ to a few $\mathrm{GeV}$, and the fluence in this range is $\sim 2 \times 10^{-5} \mathrm{ergs} \mathrm{cm}^{-2}$. Most strikingly, an additional 18 high-energy photons were recorded for $\sim 5400 \mathrm{~s}$ following this, including an $18 \mathrm{GeV}$ photon and other 36-137 MeV photons. The fluence of the delayed emission was measured to be $7 \times 10^{-6} \mathrm{ergs} \mathrm{cm}^{-2}$ in the energy range $30 \mathrm{MeV}-3 \mathrm{GeV}$. Among many models for this delayed high-energy emission from GRBs (e.g., Mészáros \& Rees 1994; Katz 1994; Plaga 1995; Totani 1998a, 1998b; Wang, Dai, \& Lu 2002), one is that the delayed emission is the result of the electromagnetic cascade of the TeV photons and the inverse Compton scattering of the CMB radiation (Cheng \& Cheng 1996).

Although this model suggests that soft photons tend to have larger amounts of delay time, a very long observation time is needed to detect this effect for delayed photons with energy $\lesssim 100 \mathrm{MeV}$, as shown by expressions of $\tau_{3}$. For GRB 940217 , the lack of high-energy photons within 36-137 MeV should occur at around $10^{5}-10^{6} \mathrm{~s}$ after the $\mathrm{keV}$ burst, while the observation of EGRET takes only $\sim 5000 \mathrm{~s}$. Thus, no correlation between time delay and photon energy could be found in the EGRET observations.

In this model, the 36-137 MeV photons should come from the cascade process of photons with energies $\mathcal{E}_{\gamma}<0.5 \mathrm{TeV}$. Since this part of high-energy photons may not be totally absorbed by IR photons, especially if this burst is at a low redshift, we get $f \gtrsim 0.01$ for this burst. Up to the detection time of GRB 940217, EGRET has had some exposure to about 150 BATSE bursts (Hurley et al. 1994). Since GRB 940217 was the third strongest one of $\sim 800$ BATSE bursts and had a fluence of $(6.6 \pm 2.7) \times 10^{-4} \mathrm{ergs} \mathrm{cm}^{-2}$, we estimate that only $\sim 1$ burst with fluence $F \gtrsim 2 \times 10^{-4} \mathrm{ergs} \mathrm{cm}^{-2}$ had been exposed by EGRET. If $f \sim 0.01$, then only $\sim 1$ burst will have delayed emission sufficiently strong to be detected by EGRET, which is consistent with the lone detection of the delayed emission from GRBs by EGRET.

In the energy range $\epsilon=200 \mathrm{MeV}-20 \mathrm{GeV}$, there is only one delayed photon detected from GRB 940217, and therefore we have no reliable photon spectrum for the delayed emission of 
this energy range. Below this energy, the delayed photon spectrum may deviate from the form equation (10), as the original very high-energy photons corresponding to this part of delayed emission may be partially absorbed by the CIB. Moreover, the expected theoretical spectrum (eq. [10]) is a time integrated spectrum over the whole duration of the delayed emission, while GRB 940217 was observed in a limited time. So we could not constrain the origin of its $\mathrm{TeV}$ photons by comparing the form of equation (10) with the photon spectrum of the delayed emission from GRB 940217, although a numerical approach taking account of the gammaray absorption effect might be feasible. However, we expect that this method has a promising prospect in the future GLAST era because of the significantly increased sensitivity of this detector, with the delayed emission detected from many more GRBs and the spectrum of photons $\epsilon \gtrsim 200 \mathrm{MeV}$ well determined.

\section{SUMMARY}

In previous papers (Cheng \& Cheng 1996; Dai \& Lu 2002), we have suggested that the very high-energy photons from cosmological GRBs may collide with cosmic IR background photons, leading to electron/positron pair production. Inverse Compton scattering of the pairs off CMB photons will produce delayed $\mathrm{MeV}-\mathrm{GeV}$ emission. In this paper, we extend our previous works by the following points:

1. We suggest that $\mathrm{TeV}$ photons could also come from GRB external shocks. Compared with internal shocks, $\mathrm{TeV}$ photons from external shocks suffer little attenuation due to pair production with softer photons in the bursts.

2. There are a few emission models suggested for the $\mathrm{TeV}$ photons from GRBs, such as the proton-synchrotron radiation, the electron IC emission from external reverse shocks, and electron IC emission from intern shocks or external forward shocks. In this paper, we suggest that the spectrum of the delayed emission resulted from the $\mathrm{TeV}$ photons that were totally absorbed locally by the intergalactic IR background radiation could help to constraint the emission model of $\mathrm{TeV}$ photons from GRBs. Because this part of $\mathrm{TeV}$ photons are absorbed locally, the spectrum of the delayed emission is independent of the poorly known CIB. Since our treatment here is mainly analytic, a further numerical study to confirm this idea might be useful and necessary.

3. The time delay could be caused by the angular spreading effect of the scattered microwave photons or deflection of the secondary pairs due to IGMF. We present a more accurate calculation of the delay time caused by the angular spreading effect of the secondary electrons by considering recent observations of the extragalactic IR background and the theoretical prediction of the high-redshift IR background. By examining the dependence of the delay time on the photon energy, i.e., $\Delta t(\varepsilon) \propto \varepsilon^{-\delta}$, one can tell which of the two timescales is responsible for the delay time. For the delay time caused by the angular spreading effect, $\delta \simeq 1$, while $\delta=2.5$ otherwise.

Finally, we would like to point out that this model predicts a roughly constant flux for the delayed emission over the whole delay time and that soft photons tend to have larger amounts of delay, which constitute distinguished features to differentiate our model from other models.

We are grateful to the referee for his/her valuable comments and suggestions that significantly improved the paper. This work was supported by the Special Funds for Major State Basic Research Projects, the National 973 Project, the National Natural Science Foundation of China under grants 10233010 and 10221001, the Nanjing University Talent Development Foundation, and an RGC grant of the Hong Kong government.
Aharonian, F., et al. 2002, A\&A, 384, L23

Amenomori, M., et al. 1996, A\&A, 311, 919

Atkins, R., et al. 2000, ApJ, 533, L119

Baring, M. G., \& Harding, A. K. 1997, ApJ, 491, 663

Blumenthal, G. R., \& Gould, R. J. 1970, Rev. Mod. Phys., 42, 237

Böttcher, M., \& Dermer, C. D. 1998, ApJ, 499, L131

Cheng, L. X., \& Cheng, K. S. 1996, ApJ, 459, L79

Coppi, P. S., \& Aharonian, F. A. 1999, Astropart. Phys., 11, 35

Costamante, L., Aharonian, F., Ghisellini, G., \& Horns, D. 2003, NewA Rev., 47,677

Dai, Z. G., \& Lu, T. 2001, ApJ, 551, 249 2002, ApJ, 580, 1013

Dai, Z. G., et al. 2002, ApJ, 580, L7

De Jager, O. C., \& Stecker, F. W. 2002, ApJ, 566, 738

Dermer, C. D., Böttcher, M., \& Chiang, J. 1999, ApJ, 515, L49

Gehrels, N., \& Michelson, P. 1999, Astropart. Phys., 11, 277

Guetta, D., \& Granot, J. 2003, ApJ, 585, 885

Hurley, K., et al. 1994, Nature, 371, 652

Katz, J. I. 1994, ApJ, 432, L27

Kulsrud, R. 1999, ARA\&A, 37, 37

Kulsrud, R., Cowley, S. C., Gruzinov, A. V., \& Sudan, R. N. 1997, Phys. Rep., 283, 213

Lithwick, Y., \& Sari, R. 2001, ApJ, 555, 540

Madau, P., \& Phinney, E. S. 1996, ApJ, 456, 124

Mészáros, P., \& Rees, M. J. 1994, MNRAS, 269, L41

Paczyński, B., \& Xu, G. 1994, ApJ, 427, 708

\section{REFERENCES}

Padilla, L., et al. 1998, A\&A, 337, 43

Plaga, R. 1995, Nature, 374, 430

Poirier, J., et al. 2003, Phys. Rev. D, 67, 042001

Rees, M. J., \& Mészáros, P. 1992, MNRAS, 258, P41 1994, ApJ, 430, L93

Salamon, M. H., \& Stecker, F. W. 1998, ApJ, 493, 547

Sari, R., \& Esin, A. A. 2001, ApJ, 548, 787

Sari, R., \& Piran, T. 1999, ApJ, 520, 641

Sigl, G., Olinto, A. V., \& Jedamzik, K. 1997, Phys. Rev. D, 55, 4582

Sommer, M., et al. 1994, ApJ, 422, L63

Stecker, F. W., De Jager, O. C., \& Salamon, F. W. 1992, ApJ, 390, L49

Totani, T. 1998a, ApJ, 502, L13

1998b, ApJ, 509, L81

1999, MNRAS, 307, L41

Totani, T., \& Takeuchi, T. 2002, ApJ, 570, 470

Vietri, M. 1995, ApJ, 453, 883 1997, Phys. Rev. Lett., 78, 4328

Wang, X. Y., Dai, Z. G., \& Lu, T. 2001, ApJ, 556, 1010

. 2002, MNRAS, 336, 803

Waxman, E. 1995, Phys. Rev. Lett., 75, 386

Waxman, E., \& Bahcall, J. 1997, Phys. Rev. Lett., 78, 2292 2000, ApJ, 541, 707

Wright, E. 2003, preprint (astro-ph/0306058)

Wright, E., \& Johnson, B. 2001, preprint (astro-ph/0107205)

Zhang, B., \& Mészáros, P. 2001, ApJ, 559, 110 